\title{
The Genome of the Saprophytic Fungus Verticillium tricorpus Reveals a Complex Effector Repertoire Resembling That of Its Pathogenic Relatives
}

\author{
Michael F. Seidl, ${ }^{1}$ Luigi Faino, ${ }^{1}$ Xiaoqian Shi-Kunne, ${ }^{1}$ Grardy C. M. van den Berg, ${ }^{1}$ Melvin D. Bolton, ${ }^{2}$ \\ and Bart P. H. J. Thomma ${ }^{1}$ \\ ${ }^{1}$ Laboratory of Phytopathology, Wageningen University, Droevendaalsesteeg 1, 6708 PB Wageningen, The Netherlands; \\ ${ }^{2}$ United States Department of Agriculture, Agricultural Research Service, Northern Crop Science Laboratory, Fargo, North \\ Dakota 58102-2765, U.S.A.
}

Submitted 25 June 2014. Accepted 1 September 2014.

\begin{abstract}
Vascular wilts caused by Verticillium spp. are destructive plant diseases affecting hundreds of hosts. Only a few Verticillium spp. are causal agents of vascular wilt diseases, of which $V$. dahliae is the most notorious pathogen, and several $V$. dahliae genomes are available. In contrast, $V$. tricorpus is mainly known as a saprophyte and causal agent of opportunistic infections. Based on a hybrid approach that combines second and third generation sequencing, a neargapless $V$. tricorpus genome assembly was obtained. With comparative genomics, we sought to identify genomic features in $V$. dahliae that confer the ability to cause vascular wilt disease. Unexpectedly, both species encode similar effector repertoires and share a genomic structure with genes encoding secreted proteins clustered in genomic islands. Intriguingly, $V$. tricorpus contains significantly fewer repetitive elements and an extended spectrum of secreted carbohydrate-active enzymes when compared with $V$. dahliae. In conclusion, we highlight the technical advances of a hybrid sequencing and assembly approach and show that the saprophyte $V$. tricorpus shares many hallmark features with the pathogen $V$. dahliae.
\end{abstract}

Vascular wilt diseases caused by members of the fungal genus Verticillium collectively affect hundreds of plant hosts (Fradin and Thomma 2006; Klosterman et al. 2009). Genus Verticillium was recently revised to encompass only ten species of soil-borne fungi that differ in morphological features, such as their resting structures, as well as in their ability to cause plant diseases (Inderbitzin et al. 2011). Within this genus, $V$. dahliae is the most notorious plant pathogen, due to the ability to cause vascular wilt on hundreds of dicotyledonous plant species, including ecologically important plant hosts and many high-value crops (Fradin and Thomma 2006; Klosterman et al. 2009). Furthermore, V. albo-atrum, V. alfalfae, V. nonalfalfae,

The assembly is deposited in the DDBJ/EMBL/GenBank database under the accession JPET00000000 and the raw RNA-Seq sequence read is deposited under the accession SRX658508.

Michael F. Seidl and Luigi Faino contributed equally.

Corresponding author: B. P. H. J. Thomma; E-mail: bart.thomma@wur.nl

* The $\boldsymbol{e}$-Xtra logo stands for "electronic extra" and indicates that five supplementary figures and five supplementary tables are published online.

(C) 2015 The American Phytopathological Society and $V$. longisporum are also plant pathogens, albeit with narrower host ranges and more restricted distribution than $V$. dahliae (Inderbitzin and Subbarao 2014). However, not all Verticillium species are genuine plant pathogens (Inderbitzin and Subbarao 2014). For instance, although occasionally reported as an opportunistic pathogen, $V$. tricorpus is mainly known as a soil-borne saprophyte that thrives on decaying organic matter (Isaac 1967; Klosterman et al. 2009; Powell et al. 2013; Qin et al. 2008). Comparative genomics between pathogenic members of the Verticillium genus, in particular strains of $V$. dahliae, recently facilitated the identification of key factors that mediate successful establishment of host infection and mechanisms that foster adaptation in the evolutionary arms race with plant hosts (de Jonge et al. 2012; 2013; Klosterman et al. 2011; Seidl and Thomma 2014). To further identify genomic differences within the Verticillium genus and to identify genetic components that enable particular Verticillium species to successfully infect plant hosts, determination of the genome sequence of nonpathogenic members of the Verticillium genus, such as $V$. tricorpus, is instrumental.

In the past, de novo sequencing of complex eukaryotic genomes was expensive and time-consuming. However, recent advances in next-generation sequencing technologies, which can now be divided into second and third generation, facilitate affordable, rapid, and high-quality genome sequencing (Faino and Thomma 2014; Metzker 2010). Second generation sequencing generates billions of short strings of nucleotides (called reads) of high quality and can be used for genome resequencing, in which genome assemblies are based on mapping of reads to a reference genome, as well as for de novo genome sequencing. However, de novo assemblies that are based on short reads are obstructed by repetitive elements, GC- or AT-rich stretches, and homo-nucleotide stretches. Consequently, genome assemblies based only on second generation sequencing generally contain many gaps, leading to fragmented draft genomes (Faino and Thomma 2014). Third generation technology, such as single molecule real-time (SMRT) sequencing, generates long continuous sequences (up to $35 \mathrm{~kb}$ ) that overcome assembly problems by increasing the probability of having unique overlaps between reads (Faino and Thomma 2014; Schadt et al. 2010). However, SMRT sequencing has a high per-base error rate that can only be corrected by large sequence depths, which results in considerable sequencing costs. To overcome the specific limitations of second and third generation sequencing platforms, hybrid approaches have been developed (Faino and Thomma 2014; Gnerre et al. 2011; Koren et al. 2012). Such 
approaches combine reads generated by second and third generation sequencing platforms to maximize continuity and minimize the per-base error rate. So far, however, hybrid approaches have only been used for relatively small microbial (bacterial) genomes (Koren et al. 2012).

Here, we report on the genome of the saprophyte $V$. tricorpus. We applied a recently described hybrid assembly pipeline (Faino and Thomma 2014) to establish a draft genome of V. tricorpus. This $36-\mathrm{Mb}$ genome assembly encompasses 58 nearly gapless scaffolds, of which four represent complete chromosomes. Comparative genomics between $V$. tricorpus and $V$. dahliae was performed in an attempt to identify genomic features that foster pathogenicity within the Verticillium genus.

\section{RESULTS AND DISCUSSION}

\section{V. tricorpus MUCL9792}

\section{does not cause wilt disease on tomato.}

In order to confirm the inability of $V$. tricorpus to cause Verticillium wilt disease on tomato (Solanum lycopersicum), inoculations were carried out with V. tricorpus MUCL9792 on 'MoneyMaker' tomato plants that lack any characterized source of Verticillium wilt resistance (Fradin et al. 2009). As controls, inoculations were performed with two $V$. dahliae strains, VdLs17 and JR2, that have previously been characterized as relatively weak and strong tomato pathogens, respectively (de Jonge et al. 2013; Fradin et al. 2009; Klosterman et al. 2009). As expected, inoculation of tomato plants with $V$. dahliae JR2 resulted in clear stunting of the inoculated plants as a typical symptom of Verticillium wilt disease, whereas inoculation with strain VdLs17 resulted in considerably fewer disease symptoms, as only mild stunting was observed (Fig. 1A). In contrast, inoculation of tomato plants with $V$. tricorpus did not lead to visible stunting of tomato plants (Fig. 1A). This was quantitatively corroborated as plants inoculated with $V$. dahliae strains JR2 or VdLs17 show considerably reduced foliage area when compared with mock-inoculated plants, while plants inoculated with $V$. tricorpus do not show any reduction (Fig. 1B). Moreover, fungal recovery assays using stem sections of the inoculated plants showed that all sections of $V$. dahliae-inoculated plants were colonized by fungus, while no fungal biomass was recovered from stem sections of $V$. tricorpusinoculated plants (Fig. 1C). Finally, real-time polymerase chain reaction (PCR) quantification of fungal biomass revealed that no $V$. tricorpus biomass could be recorded in inoculated tomato plants, while ample $V$. dahliae biomass could be detected in VdLs17- and JR2-inoculated plants (Fig. 1D). These data confirm previous observations that $V$. tricorpus is not a genuine tomato pathogen (Isaac 1967). V. tricorpus has only incidentally been reported as a plant pathogen on other hosts (Powell et al. 2013; Qin et al. 2008). For instance, inoculation of lettuce with $V$. tricorpus resulted in mild disease symptoms on only two out of 400 assessed lettuce plants (Qin et al. 2008). Thus, V. tricorpus should be considered an opportunistic pathogen that causes occasional infections on weakened plant hosts. Indeed, $V$. tricorpus is able to induce wilt symptoms when plants have been challenged with additional abiotic stresses, such as high soil nitrogen levels or waterlogging conditions (Isaac 1956).

\section{A high-quality $\boldsymbol{V}$. tricorpus genome assembly.}

The genome sequence of $V$. tricorpus MUCL9792 was determined using sequencing-by-synthesis on the Illumina HiSeq2000 and SMRT sequencing on the PacBio platform. To this end, $1.2 \mathrm{~Gb}$ of paired-end (PE) library-derived reads (500-bp insert size; 100-bp read length) was produced in combination with $1 \mathrm{~Gb}$ of mate-pair (MP) library-derived reads (5-kb insert size; 50-bp read length), together with approximately $200 \mathrm{Mb}$

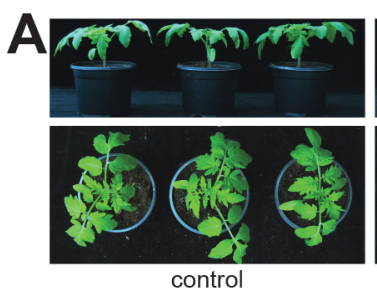

B

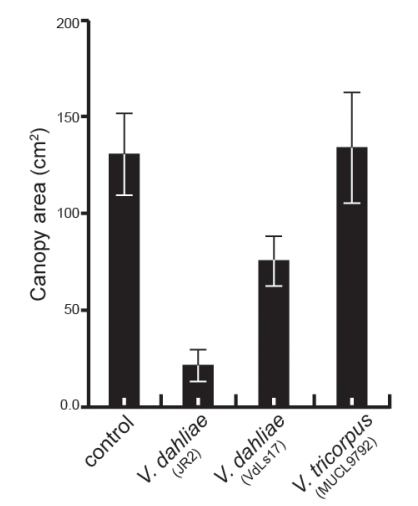

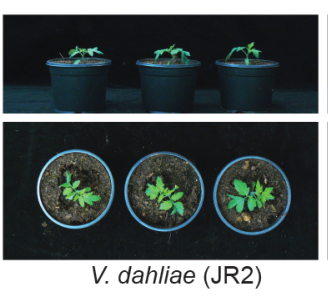

C

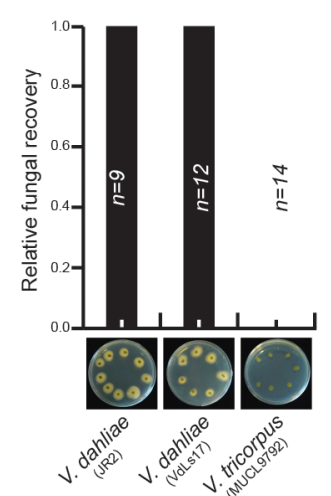

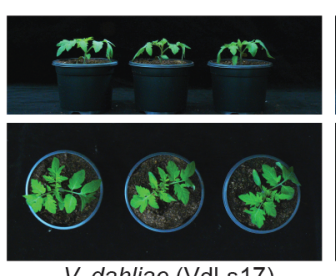

V. dahliae (VdLs17)

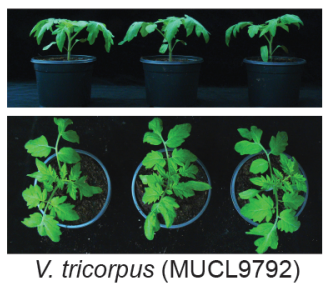

D

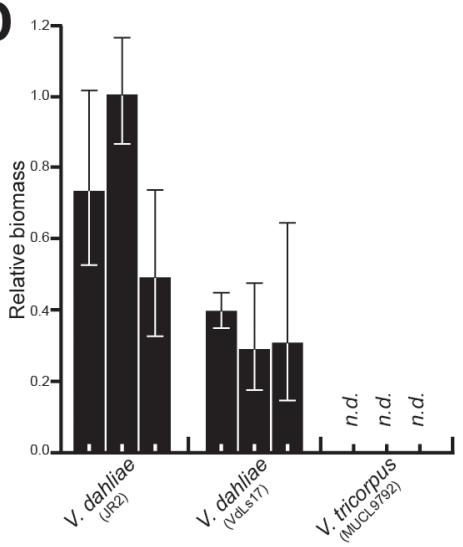

Fig. 1. Verticillium tricorpus does not cause wilt disease on tomato. Pathogenicity of two V. dahliae strains (highly pathogenic JR2, mildly pathogenic VdLs17) and of $V$. tricorpus was assessed on tomato. A, Side and top view of tomato plants at 13 days after mock inoculation (control) or inoculation with $V$. dahliae JR2, V. dahliae VdLs17, and V. tricorpus MUCL9792. B, Average canopy area of six tomato plants at 13 days after mock inoculation or inoculation with $V$. dahliae JR2, V. dahliae VdLs17, and V. tricorpus MUCL9792. An independently performed inoculation resulted in highly similar results (squared Pearson correlation coefficient $=0.99$ ). C, Fungal outgrowth at 10 days after plating tomato stem sections harvested at 15 days postinoculation with $V$. dahliae JR2, V. dahliae VdLs17, and V. tricorpus MUCL9792. Quantification shows the percentage of stem section slices that showed fungal outgrowth. D, Realtime polymerase chain reaction quantification of fungal biomass based on tomato plants harvested at 15 days postinoculation with $V$. dahliae JR2, $V$. dahliae VdLs17, and V. tricorpus MUCL9792. 
of long reads produced with PacBio sequencing (10-kb insert size) (Supplementary Table S1). In order to assemble the Illumina-derived short reads and the PacBio-derived long reads, we applied a recently described hybrid assembly approach (Fig. 2A) (Faino and Thomma 2014) that increases contiguity of the assembly in several steps (Fig. 2B). In the first step, the $\mathrm{PE}$ and MP sequences were assembled using the A5 pipeline (Tritt et al. 2012) (Fig. 2A). This initial assembly resulted in 195 scaffolds with an N50 of approximately $4 \mathrm{Mb}$, with the longest contig of $240 \mathrm{~kb}$ and the longest scaffold of $5.3 \mathrm{Mb}$
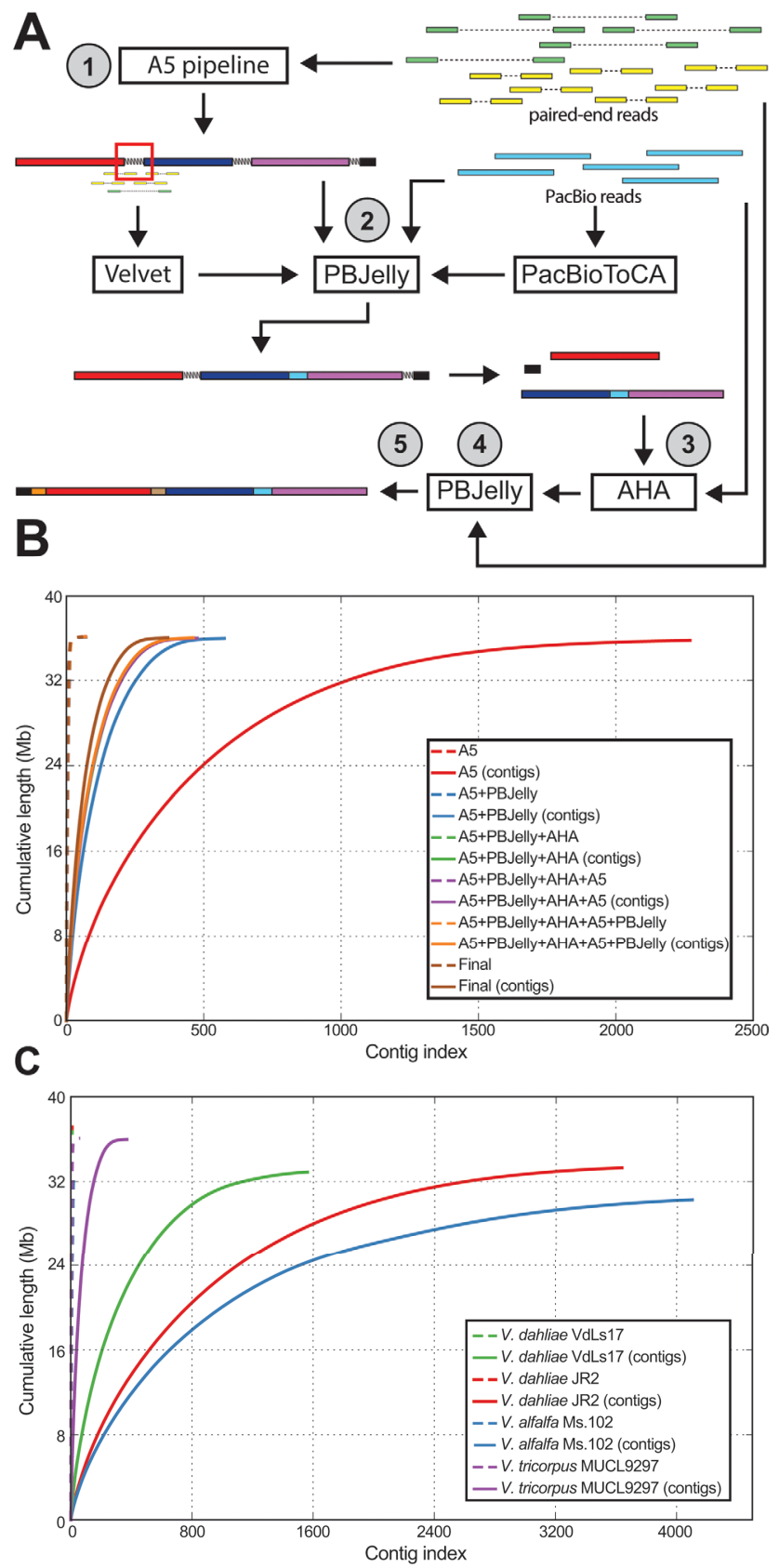

Fig. 2. A hybrid sequencing and assembly approach yields a nearly gapless draft genome of Verticillium tricorpus. A, A five-step hybrid assembly approach applied to assemble the 36-Mb draft genome of V. tricorpus. B, Sequence length accumulation curve showing the successive reduction in the number of contigs and scaffolds during the different steps of the hybrid assembly. The curve displays the number of bases in the assembly as a function of the (sorted) number of sequences (contigs or scaffolds) $\mathbf{C}, \mathrm{Se}$ quence length accumulation curve showing the comparison between the draft genome assembly of $V$. tricorpus and the previously assembled genomes of $V$. dahliae strains JR2 and VdLs17 as well as V. alfalfae.
(Table 1). This assembly has approximately 2,500 gaps and approximately $600 \mathrm{Ns}$ (unknown nucleotides) per $100 \mathrm{~kb}$. In the second step, gaps were filled using three types of sequences: i) corrected long reads, ii) uncorrected long reads, and iii) sequences generated upon local reassembly of short reads at gap positions (Fig. 2A). This step improved the assembly statistics considerably, reducing the number of contigs from approximately 2,700 to approximately 750 , with the longest contig of $535 \mathrm{~kb}$, and reducing the number of gaps from approximately 2,500 to approximately 550. At this stage, gaps that are not closed may actually represent wrongly scaffolded contigs. Therefore, uncorrected long reads were used to scaffold contigs in the third step. Subsequently, the obtained scaffolds were subjected to another scaffolding step, using MP reads in the fourth step, resulting in 604 contigs and 166 scaffolds (Table 1). More importantly, the total number of gaps was reduced to 438, while the number of Ns per $100 \mathrm{~kb}$ dropped to approximately 240. Manual curation included the removal of about 100 extremely short contigs $(<500 \mathrm{bp})$ and further scaffolding. The A5 software will not merge contigs into scaffolds in case of insufficient support by paired Illumina reads. However, in about 10 cases in which MP reads could unambiguously be mapped at the edges of two contigs, these were joined manually. Subsequently, we globally evaluated the scaffolding by remapping the MP reads to the scaffolds. As this did not lead to the identification of any discordantly mapped MP reads that would suggest erroneous scaffolding, this assembly was considered final (Supplementary Fig, S1). This manual curation yielded a final assembly of 399 contigs that composed 61 scaffolds (58 genomic and three mitochondrial) ranging in size between $9 \mathrm{Mb}$ and $500 \mathrm{bp}$ (scaffold N50, $4.7 \mathrm{Mb}$; contig N50, $250 \mathrm{~kb}$ ) with 338 gaps and $229 \mathrm{Ns}$ per $100 \mathrm{~kb}$, on average (Table 1). The 10 largest scaffolds encompass $95 \%$ of the genome (Figs. 2C and 3A).

To determine its quality, the genome assembly was compared with that of previously sequenced Verticillium genomes. The genome of V. dahliae VdLs17 as well as that of V. alfalfae (formerly known as $V$. albo-atrum) VaMs102 were sequenced using whole-genome shotgun Sanger sequencing $(7.5 \times$ and $4 \times$ coverage, respectively) (Klosterman et al. 2011), while $V$. dahliae JR2 was sequenced with the Illumina HiSeq2000 (30× coverage) (de Jonge et al. 2012). All these assemblies yielded a higher number of contigs (1,562 for $V$. dahliae VdLs17, 4,098 for $V$. alfalfae, and 4,515 for $V$. dahliae JR2) when compared with the V. tricorpus assembly (Fig. 2C), leading to a considerably higher number of Ns per $100 \mathrm{~kb}$. Consequently, the scaffolded genome assembly of $V$. alfalfae and $V$. dahliae VdLs17 contained 2.5 and $0.9 \mathrm{Mb}$ of $\mathrm{Ns}$, respectively. Although optical mapping further refined the $V$. dahliae assemblies by combining individually assembled scaffolds into eight chromosomes (de Jonge et al. 2013; Klosterman et al. 2011), this process introduced a considerable number of additional gaps.

To assess whether any of the 58 scaffolds of the V. tricorpus assembly represent full-length chromosomes, we queried the scaffolds for characteristic fungal telomeric sequence repeats (Fulnecková et al. 2013). We identified 14 such repeats, all of which were located at the ends of scaffolds (Fig. 3A). Moreover, eight telomeric repeats were found on the ends of four of the 10 largest scaffolds that are, thus, likely to represent fulllength chromosomes (Fig. 3A). Pulse-field gel electrophoresis was performed to determine the karyotype of $V$. tricorpus MUCL9792. This analysis revealed the presence of seven chromosomes, suggesting that we sequenced and assembled all telomeres present in the genome (Fig. 3B). Combining the information from the karyotyping and the assembly, we estimate the sizes of the chromosomes to be $9 \mathrm{Mb}$ (Scaffold 1; not entered the gel), 7.5 Mb (Scaffold 2), 5.7 Mb (not completely 
assembled), 4.8 Mb (not completely assembled), 4.1 Mb (Scaffold 4), 3.3 Mb (Scaffold 5), and 1.8 Mb (not completely assembled) in size (Fig. 3B), summing up to $36.2 \mathrm{Mb}$ and corresponding to the calculated genome size. Thus, whereas previously sequenced $V$. dahliae strains carry eight chromosomes, this $V$. tricorpus strain only carries seven.

To further assess the completeness of the assembled gene space, we queried for orthologs of 248 core eukaryotic gene families, using the CEGMA pipeline (Parra et al. 2007, 2009). The assembled $V$. tricorpus genome assembly was found to contain 237 members $(95.56 \%)$ of these eukaryotic core families, comparable to previously assembled $V$. dahliae draft genomes and higher when compared with $V$. alfalfae (81\%) (de Jonge et al. 2013; Klosterman et al. 2011), indicative of a highly complete gene space in the $V$. tricorpus genome assembly.

\section{V. tricorpus genome annotation.}

We classified different types of repetitive elements in the genome of $V$. tricorpus with Repeatmasker, utilizing a combination of known repetitive elements and de novo repeat identification (Jurka et al. 2005; Smit et al. 1996). In total, $2.5 \%$ $(800 \mathrm{~kb})$ of the assembled $V$. tricorpus genome accounts for repetitive DNA and included traces of long terminal repeats $(110 \mathrm{~kb}, 0.3 \%)$, long interspersed nuclear elements $(35 \mathrm{~kb}$,

Table 1. Genome assembly statistics for the A5 assembly, the hybrid assembly, and the final assembly of the draft genome of Verticillium tricorpus

\begin{tabular}{lccc}
\hline Statistics & A5 & Hybrid assembly & Final assembly \\
\hline Final statistics & & & \\
$\quad$ Read depth (fold) & $62.1 \mathrm{X}$ & $67.5 \mathrm{X}$ & $67.5 \mathrm{X}$ \\
Estimate genome size (Mb) & 36.16 & 36.10 & 36.09 \\
Number of Ns (unknown nucleotides) & 217,012 & 87,959 & 82,887 \\
Number of Ns per 100 kb & 600.20 & 242.65 & 229.6 \\
Contig statistics & & & \\
Number & 2,789 & 604 & 399 \\
Total length (Mb) & 35.94 & 36.04 & 36.01 \\
Largest (kb) & 241.5 & 677.2 & 677.2 \\
$\mathrm{~N}_{50}(\mathrm{~kb})$ & 35.9 & 180.7 & 249.2 \\
Number of genes (partial) & $10,417(1,032)$ & $11,291(167)$ & $11,338(120)$ \\
Scaffold statistics & 195 & & \\
Number & 5,284 & 166 & 61 \\
Largest (kb) & 4,123 & 6,960 & 9,084 \\
$\mathrm{~N}_{50}(\mathrm{~kb})$ & 600.71 & 4,117 & 243.65 \\
Number of Ns per 100 kb & $11,107(345)$ & $11,398(60)$ & 229.64 \\
Number of genes (partial) & & & $11,406(52)$ \\
\hline
\end{tabular}

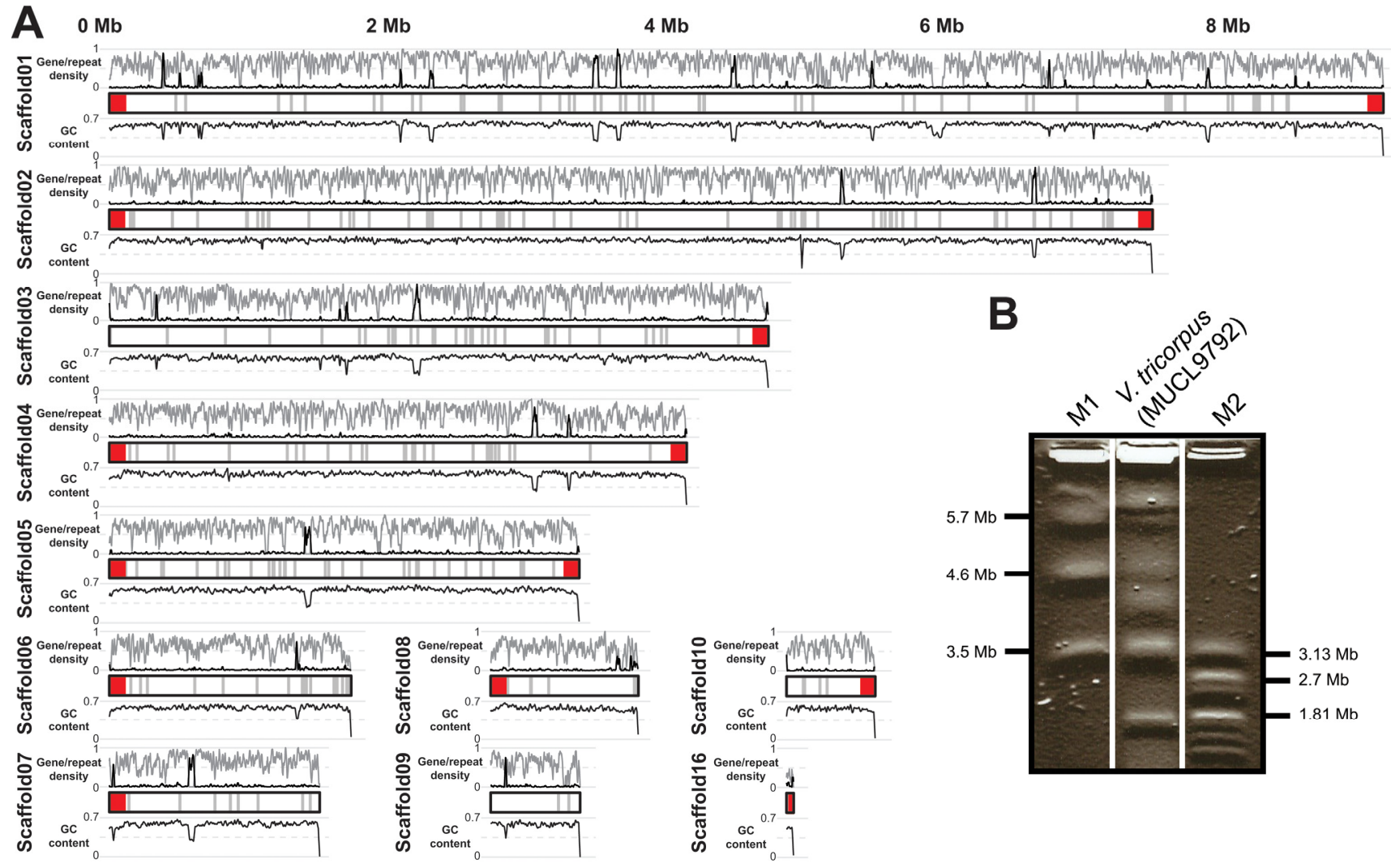

Fig. 3. Overview of the draft genome assembly of Verticillium tricorpus. A, Schematic representation of the 10 largest scaffolds in the genome assembly of $V$. tricorpus. Characteristic fungal telomeric repeats are displayed on the ends of the scaffolds (indicated by shading; an additional single small scaffold with distal telomeric repeat is displayed as well), and assembly gaps are displayed on each scaffold. Gene (gray) and repeat (black) density as well as GC content is displayed in a sliding window of $10 \mathrm{~kb}$ with a slide of $2 \mathrm{~kb}$. B. Chromosome karyotype by pulsed-field gel electrophoresis of the sequenced $\mathrm{V}$. tricorpus MUCL9792. Chromosomal DNA of Schizosaccharomyces pombe (M1) and Hansenula wingei (M2) were loaded as size markers. 
$0.1 \%$ ), and short interspersed nuclear elements ( $1 \mathrm{~kb}, 0.002 \%)$ that are not uniformly distributed along the scaffolds (Fig. 3A). Surprisingly, their quantity and relative contribution is considerably smaller compared with that of previously sequenced Verticillium genomes, in which $4.2 \%$ of the assembled genome account for repetitive elements $(4.05 \%$ for $V$. dahliae VdLs 17 and $4.33 \%$ for V. dahliae JR2) (de Jonge et al. 2013; Klosterman et al. 2011). Repetitive elements are strong drivers of genome evolution, since they promote large-scale genomic variations (Seidl and Thomma 2014). In V. dahliae, repetitive elements have been shown to foster genomic rearrangements that drive the evolution of pathogenicity (de Jonge et al. 2013; Seidl and Thomma 2014). It is, thus, tempting to speculate that the increase of repetitive elements in the genome of $V$. dahliae is associated with its pathogenic capacity.

We inferred a reference gene annotation by integrating de novo, homology-based, and transcription data using the Maker2 pipeline (Holt and Yandell 2011). To this end, we generated 1-Gb RNA-Seq data on $V$. tricorpus growing in a suspension of tomato cells, to mimic a plant-associated environment, and about $97 \%$ of the reads could be aligned to the genome assembly using TopHat2 (Kim et al. 2013). To further improve gene structure annotation by Maker2, we assembled the RNA-Seq reads into transcripts, using Oases (Schulz et al. 2012) and Trinity (Grabherr et al. 2011), which were subsequently aligned to the genome assembly using PASA (Haas et

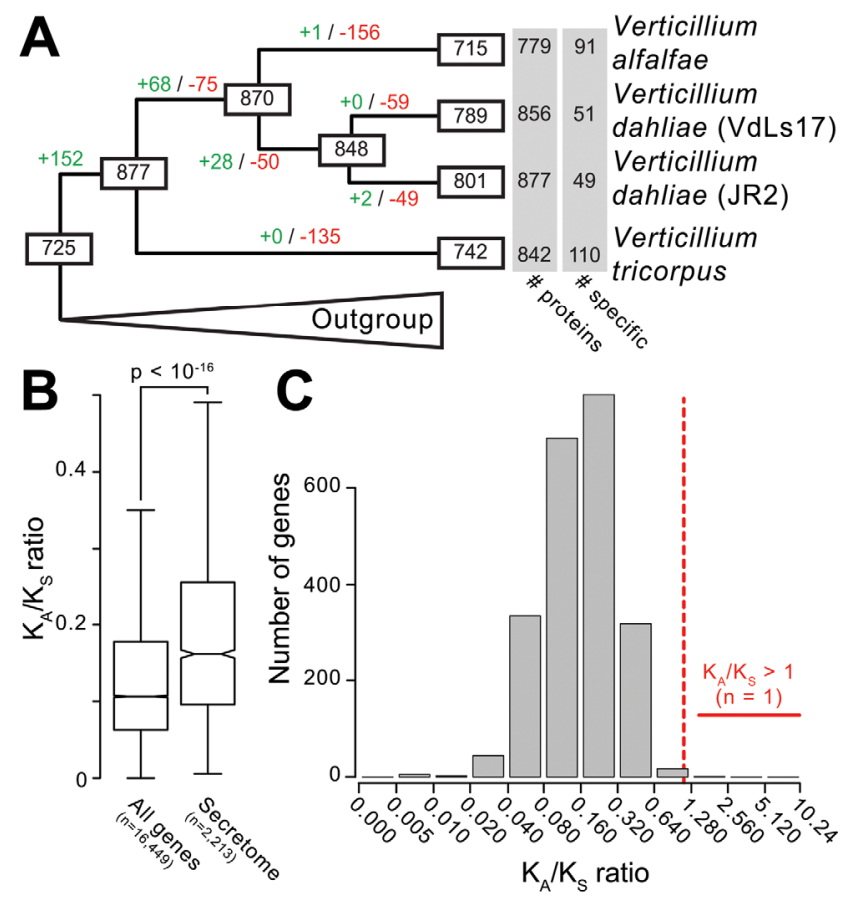

Fig. 4. The secretome of Verticillium tricorpus encodes a secretome of similar size as pathogenic Verticillium spp. A, Evolutionary history of the gene families constituting the predicted secretome of the analyzed Verticillium spp. according to DOLLOP. At each branch, the number of gene family gains and losses is displayed and the inferred ancestral gene family set is shown. B, Distribution of nonsynonymous substitutions per nonsynonymous site $\left(\mathrm{K}_{\mathrm{A}}\right)$ to synonymous substitutions per synonymous site $\left(\mathrm{K}_{\mathrm{S}}\right)$ ratios for $V$. tricorpus genes encoding secreted proteins with orthologs in any of the analyzed Verticillium secretomes and for all other $V$. tricorpus genes with orthologs in any of the analyzed Verticillium species.. The difference between the distributions was assessed using the nonparametric Wilcoxon rank sum test and significance is highlighted. Whiskers indicate the 1.5 interquartile range and outlier were not displayed for clarity. The total number of considered gene pairs $(n)$ is indicated. C, Distribution of $\mathrm{K}_{\mathrm{A}} / \mathrm{K}_{\mathrm{S}}$ ratios for $V$. tricorpus genes encoding secreted proteins with orthologs in the other analyzed Verticillium secretomes. Genes with $K_{A} / K_{S}$ ratios $>1$, indicative of positive selection, are highlighted. al. 2003). Additionally, we provided Maker2 (Holt and Yandell 2011) with 35 predicted fungal proteomes that represent a broad phylogenetic diversity (Supplementary Table S2) to guide gene structure annotation and applied three independent de novo gene predictors: SNAP (Korf 2004), GeneMark-HMM (Lukashin and Borodovsky 1998), and Augustus (Stanke et al. 2004). Maker 2 combined these data and produced a consolidated set of gene models. Subsequently, we refined these gene models by manually performing RNA-Seq- and homologyguided gene structure annotation. If necessary, gene models were corrected, leading to the identification of 418 previously undetected genes, mainly due to erroneous gene fusions, and the correction of approximately 4,000 gene models, thus yielding a total of 11,454 protein-coding genes. Of these, $89 \%$ have RNA-Seq support ( $\geq 1$ read mapped, on average 464 reads per gene model) and $91 \%$ have homology-based support in other fungal species. The 11,454 protein-coding genes were functionally annotated using InterProScan (Zdobnov and Apweiler 2001) and Blast2GO (Conesa et al. 2005), yielding approximately $65 \%$ of the predicted genes with a predicted functional annotation (Supplementary Fig. S2; Supplementary Table S3).

\section{The secretome of $V$. tricorpus.}

The vast majority of fungal species comprise saprophytes that thrive on decaying organic matter. These species deploy a plethora of secreted proteins to decompose complex organic material in their proximity to acquire nutrients and to establish their niche. Independently emerging from saprophytic ancestors, particular fungal species evolved to establish symbioses with various hosts, including plants, requiring them to successfully overcome host immunity. Irrespective of whether the symbiosis involves parasitism, commensalism, or mutualism, secreted fungal proteins play an important role, as they comprise so-called effectors that are required for establishment of the interaction (de Jonge et al. 2011; Rovenich et al. 2014; Thomma et al. 2011). Therefore, pathogenic species often encode larger secretomes (i.e., the total set of secreted proteins) as compared with their saprophytic relatives (Choi et al. 2010), potentially reflecting the acquisition of additional factors, e.g., effector genes, that facilitate their pathogenic lifestyle. To predict the secretome of $V$. tricorpus, we queried the predicted proteome for secretion signals, leading to the identification of 952 secreted proteins, which accounts for approximately $8.3 \%$ of its proteome. Using the same approach, the genomes of the pathogenic $V$. dahliae strains JR2 and VdLs17 encode 926 and 907 secreted proteins accounting for approximately $8.5 \%$ of their predicted proteomes, respectively. The genome of $V$. alfalfae encodes fewer (870) secreted proteins, which may be the consequence of the relatively low sequence coverage that hampered whole-genome assembly and annotation (Klosterman et al. 2011) and, thus, should be taken with caution.

By tracing the gain and losses of gene families encoding secreted proteins in the Verticillium genus using Dollo parsimony, we observed comparable absolute numbers of these gene families in $V$. dahliae, $V$. alfalfae, and $V$. tricorpus ranging from 715 to 801 , corresponding to 779 to 877 proteins (Fig. 4A). Since pathogenic Verticillium species likely evolved from a nonpathogenic saprophytic ancestor, the 667 families (2,691 proteins) that are shared between $V$. tricorpus, $V$. dahliae, and $V$. alfalfae could represent a 'core' saprophytic secretome. In $V$. dahliae JR2, only 56 of the 668 genes belonging to these families are in planta induced (fold change $>1.5 \log _{10}$ ), and thus, the majority have other functions that are likely not associated with its pathogenic lifestyle. Only a few gene families (99) encompassing 254 genes encoding secreted proteins have been specifically gained in the genomes of $V$. dahliae and $V$. alfalfae, of which the vast majority encode proteins of unknown function. Moreover, a consider- 
able amount of secreted proteins do not display similarity to any other sequence and are thus considered lineage-specific. For example, $V$. dahliae JR2 contains 49 lineage-specific genes encoding secreted proteins, including the experimentally verified virulence effector XLOCO09059 and the in planta-induced candidate effector XLOC008951 (de Jonge et al. 2013). Similarly, $V$. tricorpus encodes a considerable amount of lineagespecific genes, mainly encoding secreted proteins with unknown function. The vast majority $(70 \%)$ of these genes are expressed during in vitro growth.

Many genes encoding secreted proteins, but particularly pathogen effectors, are expected to rapidly diversify due to selection pressure imposed by the host during the evolutionary arms race between pathogens and their hosts (de Jonge et al. 2013; Guyon et al. 2014; Klosterman et al. 2011; Raffaele et al. 2010; Wicker et al. 2013). To assess the speed of gene evolution and positive selection within the secretome, we used the ratio of nonsynonymous substitutions per nonsynonymous site $\left(\mathrm{K}_{\mathrm{A}}\right)$ to synonymous substitutions per synonymous site $\left(\mathrm{K}_{\mathrm{S}}\right)$ for $V$. tricorpus genes with orthologs in the other analyzed Verticillium spp. as a proxy (Fig. 4B). While the vast majority of $V$. tricorpus genes were found to be under purifying selection (i.e., $\mathrm{K}_{\mathrm{A}} / \mathrm{K}_{\mathrm{S}}<1$ ), genes encoding secreted proteins were found to display significantly higher $\mathrm{K}_{\mathrm{A}} / \mathrm{K}_{\mathrm{S}}$ ratios when compared with pairs of genes derived from the full set of protein-coding genes (Fig. 4B and C), thus suggesting accelerated evolution within the secretome. However, only a single highly expressed gene, encoding a secreted protein with similarity to chitinases, displays signs of positive selection, i.e., $\mathrm{K}_{\mathrm{A}} / \mathrm{K}_{\mathrm{S}}>1$ (Fig. 4C). In contrast, $V$. dahliae JR2 contains 17 genes encoding secreted proteins with signs of positive selection (Supplementary Fig. S3), of which the majority are of unknown function, but that also includes genes encoding secreted proteins with predicted functions in cellulose binding or cutinase activity. In many pathogenic microbes, an extended repertoire of genes that are under positive selection and encode secreted proteins has been described (Raffaele et al. 2010; Wicker et al. 2013). However, the small number of genes under positive selection corroborates previous analysis that suggested that diversification at nucleotide levels does not play a major role in $V$. dahliae evolution (de Jonge et al. 2013). Notably, the majority of effector genes in $V$. dahliae with roles in virulence are lineage-specific, hampering the assessment of positive selection, and suggesting that presence or absence polymorphisms are crucial to drive the evolution of pathogenicity in $V$. dahliae.

We observed a distinct quantitative difference in repeat content between $V$. dahliae JR2 and $V$. tricorpus. To further assess the hypothesis that the expansion of repeats in $V$. dahliae JR2 is associated with its pathogenicity (de Jonge et al. 2013; Seidl and Thomma 2014), we analyzed whether genes, particularly those encoding secreted proteins, are directly flanked by repetitive elements. In V. dahliae JR2, $25 \%$ of all genes and $27 \%$ of those encoding secreted proteins are directly flanked by at least a single repetitive element (excluding simple nucleotide repeats). In contrast, only $15 \%$ of all predicted genes and $18 \%$ of those encoding secreted proteins are flanked by repeats in $V$. tricorpus. Interestingly, when considering in planta-expressed genes ( $>1.5 \log _{10}$ fold change), 20 of 79 genes that encode secreted proteins are directly flanked by repeats in $V$. dahliae JR2, including two previously functionally characterized JR2specific effector proteins (de Jonge et al. 2013). In contrast, only three highly expressed $V$. tricorpus genes $\left(>2 \log _{10}\right.$ (fold change) when compared with the average genome-wide expression) that encode secreted proteins are flanked by repeats. Thus, our data suggest that the presence and expansion of repetitive elements is associated with pathogenicity in the Verticillium genus.

\section{Genes encoding secreted proteins are clustered in Verticillium spp.}

The 10 largest scaffolds encode, on average, 9.5\% secreted proteins. On some of the smaller scaffolds, considerably more genes encode secreted proteins, e.g., up to $18 \%$ of the genes on scaffold 10, thus indicating that many of these genes localize in close physical proximity to each other. Moreover, these small scaffolds contained one of the characteristic telomeric repeats and, thus, many proteins encoding secreted protein cluster in proximity of the chromosomal ends. Therefore, we analyzed the global position of genes encoding secreted proteins on the complete chromosomes (scaffolds 1, 2, 4, and 5) (Fig. 5A). Interestingly, genes encoding secreted proteins are not randomly distributed along the chromosomes of $V$. tricorpus but, rather, cluster locally, both in areas within the chromosomes and also, in some cases, close to telomeres (Fig. 5A). Also, genes encoding predicted membrane- or nuclear-localized proteins cluster (Fig. 5A). However, compared with genes encoding secreted proteins, where approximately $45 \%$ of the genes are clustered, only about $27 \%$ of the genes encoding proteins predicted to localize in membranes or in the nucleus are located within clusters (Fig. 5B). Similarly, this local clustering, even though less pronounced, is also observed in both $V$. dahliae strains, e.g., on chromosome 1 for strain JR2 and chromosome 2 for VdLs17 (Fig. 5C and D). Clustering of genes encoding secreted proteins has been reported previously in different strains of $V$. dahliae, in which in planta-expressed effector genes are enriched in highly dynamic lineage-specific regions (de Jonge et al. 2013). Similarly, many filamentous pathogens contain a genome architecture that is often characterized by the local co-occurrence of effector genes (Raffaele and Kamoun 2012). For example, genes encoding secreted proteins in Fusarium graminearum are clustered preferentially in regions close to the telomeres or in regions with increased recombination frequency (Brown et al. 2012). Clustering of genes, particularly genes encoding secreted proteins, in or in the proximity of unstable genomic regions can foster the swift alterations of these genes, thus enabling the rapid adaptation to changing environments (Seidl and Thomma 2014).

\section{The genome of $V$. tricorpus encodes an expanded repertoire of CAZymes.}

Saprophytes and plant pathogens secrete a plethora of enzymes that are involved in the breakdown of complex polysaccharides that compose plant cell walls. Whereas saprophytes degrade these complex polysaccharides solely for nutritional purposes, pathogens need to also overcome these physical barriers to establish infection. Therefore, plant pathogens often encode larger repertoires of carbohydrate-active enzymes (CAZymes) when compared with saprophytes (Zhao et al. 2013). To explore the diversity of CAZymes in Verticillium species, we queried the predicted proteomes of V. tricorpus, V. alfalfae, and the two $V$. dahliae strains (JR2 and VdLs17) for CAZyme signature domains (Yin et al. 2012) that belong to the four catalytic classes of CAZymes-glycoside hydrolases $(\mathrm{GH})$, polysaccharide lyases, carbohydrate esterases, glycosyltransferases-and carbohydrate-binding modules (CBM), leading to the identification of 646, 508, 531, and 532 CAZymes (approximately 5\% of their proteomes), respectively. When compared with six filamentous pathogens (Supplementary Fig. S4) and three saprophytes, Verticillium spp., together with the vascular wilt pathogen Fusarium oxysporum, encode the highest absolute number of CAZymes in their genomes (Supplementary Fig. S5; Supplementary Table S4) (Klosterman et al. 2011). Moreover, Verticillium spp. devote the largest proportion of their proteome to CAZymes (Fig. 6A). Notably, the saprophytic dung-decomposing fungus Podospora anserina, the white rot fungus 
Phanerochaete chrysosporium, and Neurospora crassa encode considerably lower CAZyme numbers. Moreover, Verticillium spp. devote a large proportion (about $25 \%$ ) of their secretome to CAZymes (Fig. 6B), which is greater than most other fungi, with the exception of the saprophyte Phanerochaete chrysosporium (Fig. 6B). Contrary to previous observations that saprophytes encode fewer CAZymes than encoded by pathogens (Zhao et al. 2013), V. tricorpus contains more CAZymes than the two pathogenic $V$. dahliae strains. The high number of CAZymes in V. tricorpus is mainly due to a higher abundance of GH families (in particular families GH3, GH43, and GH78) and secreted CBM1-containing proteins. CBM1 is generally considered as a fungal cellulose-binding domain that is often identified in proteins containing GH (Klosterman et al. 2011). Saprophytic fungi, such as Podospora anserina and Phanerochaete chrysosporium, also encode an expanded repertoire of CBM1 containing genes. The expanded CAZyme repertoire in V. tricorpus, therefore, likely reflects its saprophytic lifestyle. In contrast, only six CAZyme families are expanded in the pathogenic Verticillium strains when compared with V. tricorpus. Moreover, a single member belonging to CAZyme family GH54 is present in all pathogenic Verticillium strains but is absent from V. tri- corpus. This gene is predicted to encode a secreted arabinofuranosidase with C-terminal CBM42. Interestingly, the gene that encodes this member is highly induced during in planta infection in $V$. dahliae JR2 (up to $3 \log _{10}$ (fold change) at 12 days postinoculation), suggesting a possible role of this gene during pathogenicity. However, only two other genes that belong to the six expanded CAZyme families show induced in planta expression ( $>1.5 \log _{10}$ fold change), suggesting that expansion or the presence or absence of particular CAZymes is not associated with pathogenicity.

\section{The $V$. tricorpus effector repertoire largely resembles that of other Verticillium spp.}

The surprising finding that the secretome of the saprophyte $V$. tricorpus is even larger than the secretome of the $V$. dahliae strains spurred us to further explore the presence and abundance of genes that have been implicated in fungal pathogenicity, and could, thus, reflect the adaptation of pathogenic Verticillium spp. to their particular lifestyle. We queried the genomes for genes encoding small secreted cysteine-rich proteins (SSCP) that are often characterized as lineage-specific pathogen effector molecules (de Jonge et al. 2011; Thomma et al. 2011). When
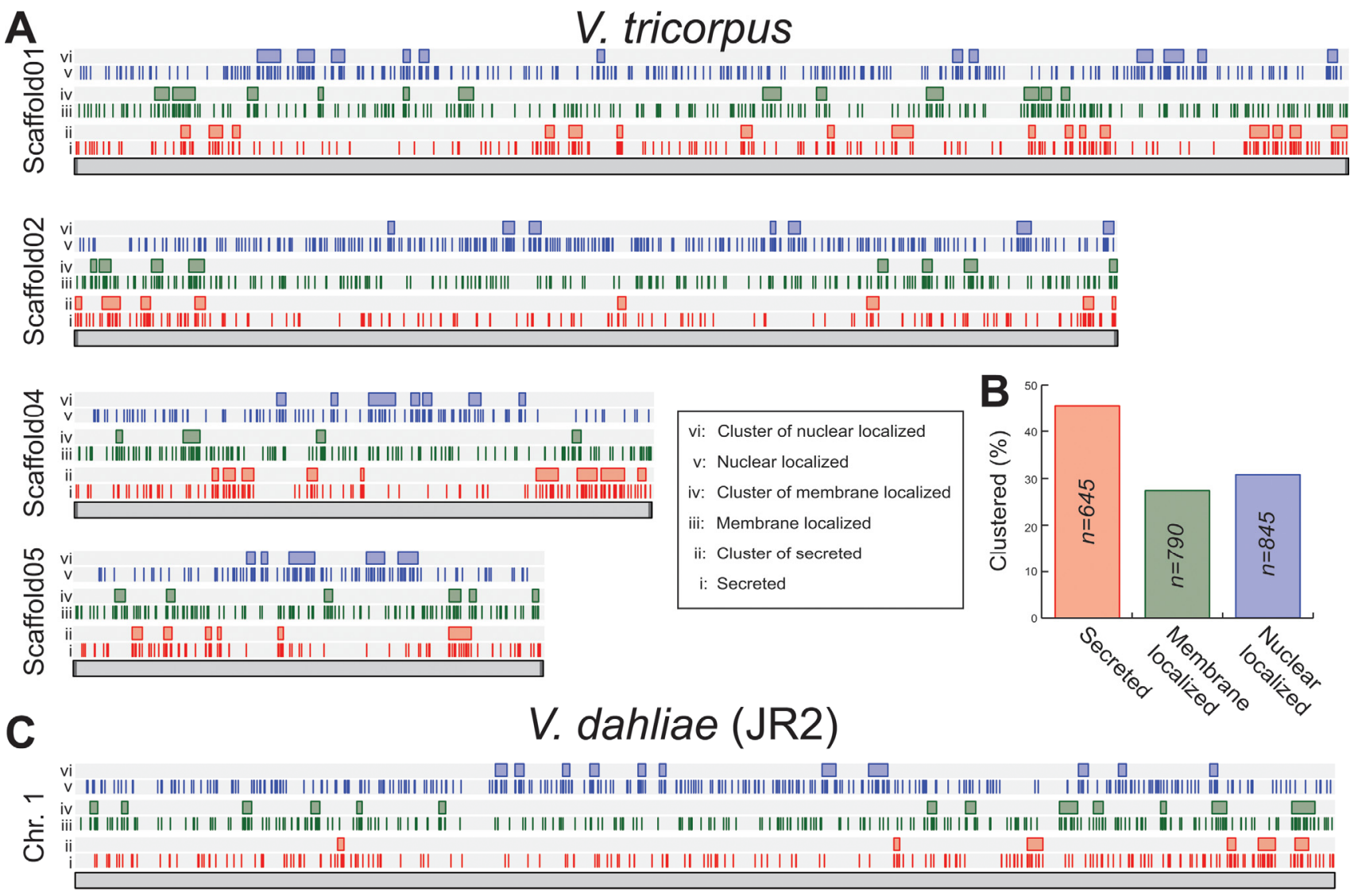

D

\section{V. dahliae (VdLs17)}

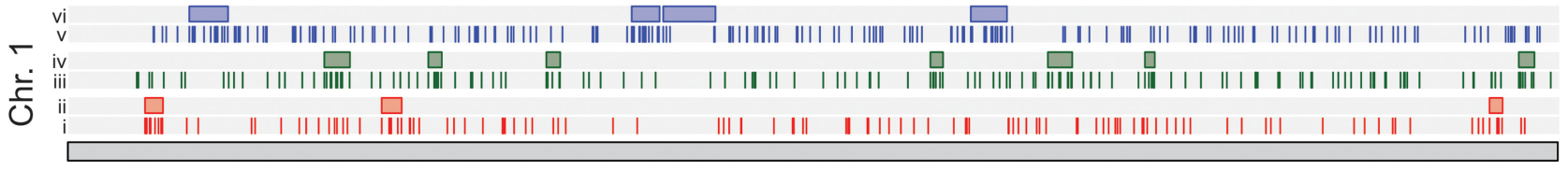

Fig. 5. Genes encoding proteins belonging to distinct functional classes cluster in the genome of Verticillium tricorpus. A, Genomic position of genes encoding secreted proteins (i), genes encoding transmembrane localized protein (iii) and genes encoding nuclear localized proteins (v) are indicated along the four scaffolds that represent complete $V$. tricorpus chromosomes. Clusters of genes for each of the functional classes are shown (ii, iv, and vi; window of 20 genes, minimum of five genes, consecutive windows were merged). B, Fraction of genes in each of the functional classes that is clustered on the four complete chromosomes. Total number of genes per class $(n)$ is indicated. C, Clustering of genes for a chromosome of $V$. dahliae JR2 and $\mathbf{D}$, VdLs 17 is shown for comparison. 
querying secreted proteins for size and cysteine content $(\leq 150$ aa and containing $\geq 3 \%$ cysteine-residues [Saunders et al. 2012]), we identified $46 \mathrm{SSCP}$ in $V$. tricorpus, of which more than $75 \%$ do not have any functional annotation. Similarly, 67 SSCP were identified in $V$. dahliae JR2, 43 in VdLs17, and 36 in V. alfalfae, of which more than 85, 70, and 70\% do not have any functional annotation, respectively (Supplementary Table S5). V. tricorpus and $V$. dahliae considerably overlap in the functionally annotated proteins present in their respective SSCP catalogue, suggesting that $V$. tricorpus shares effectors with $V$. dahliae. However, the $V$. tricorpus catalogue of SSCP contains proteins that cannot unambiguously be implicated in pathogenicity, such as hydrophobins. Even though particular hydrophobins play roles in pathogenicity (Talbot et al. 1993), they are also present in nonpathogenic fungi and act in the formation of resting structures in Verticillium spp. (Klimes et al. 2008). Similarly, several proteins with a cerato-platanin domain were identified, of which homologs occur in filamentous fungi with all types of lifestyles and that may act in in fungal growth and development (Gaderer et al. 2014).
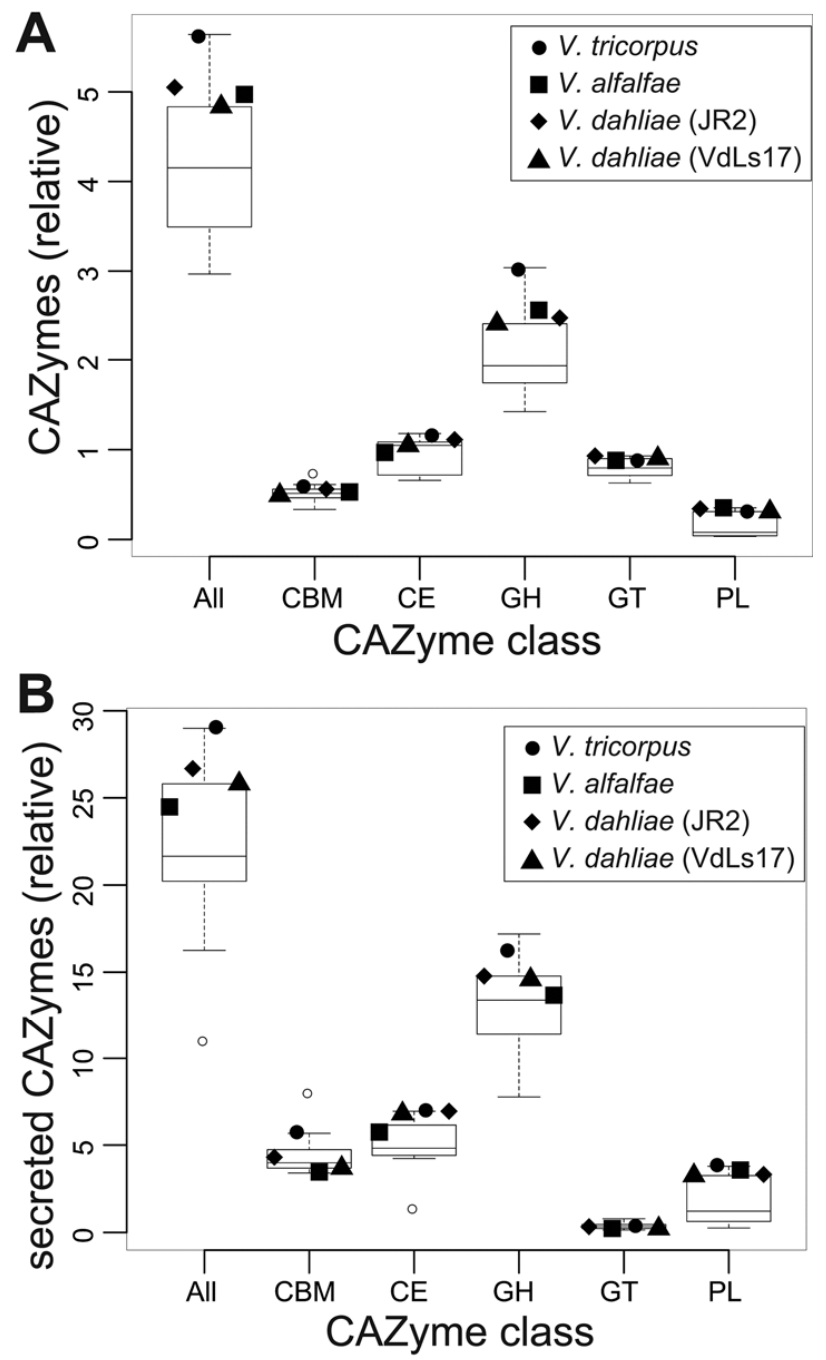

Fig. 6. The genomes of Verticillium spp. encode a large repertoire of carbohydrate active enzymes (CAZymes). A, Boxplots display the relative number of CAZymes in the full proteomes and B, secretome of Verticillium spp. and related fungi. The relative contribution belonging to the five CAZyme classes-glycoside hydrolases $(\mathrm{GH})$, polysaccharide lyases (PL), carbohydrate esterases (CE), and glycosyltransferases (GT), and carbohydrate-binding modules (CBM) - are shown as boxplots; whiskers indicate the 1.5 interquartile range, and outliers are shown as open circles. The position of each of the Verticillium spp. is indicated.
We further mined the secretomes for the presence of LysM effectors, a class of secreted proteins that do not contain any other recognizable features except LysM domains (Bolton et al. 2008; de Jonge and Thomma 2009; de Jonge et al. 2010; Kombrink and Thomma 2013). Even though several LysM effectors have been implicated in fungal pathogenicity (de Jonge et al. 2010; Marshall et al. 2011; Mentlak et al. 2012; SánchezVallet et al. 2013), they are commonly observed in saprophytes as well and may, thus, play alternative roles in fungal biology besides promoting pathogenicity (Kombrink and Thomma 2013; Rovenich et al. 2014). The genome of $V$. tricorpus encodes five LysM effectors, containing three to five LysM domains, of which one, Vt01g11890, is expressed when V. tricorpus is grown in a tomato cell suspension (Fig. 7A). Initially, seven LysM effector candidates were identified in the genome of $V$. dahliae VdLs17 (Klosterman et al. 2011), of which only five qualify as bona fide LysM effectors (Kombrink 2014). Notably, one of these five is a strain-specific LysM effector that is

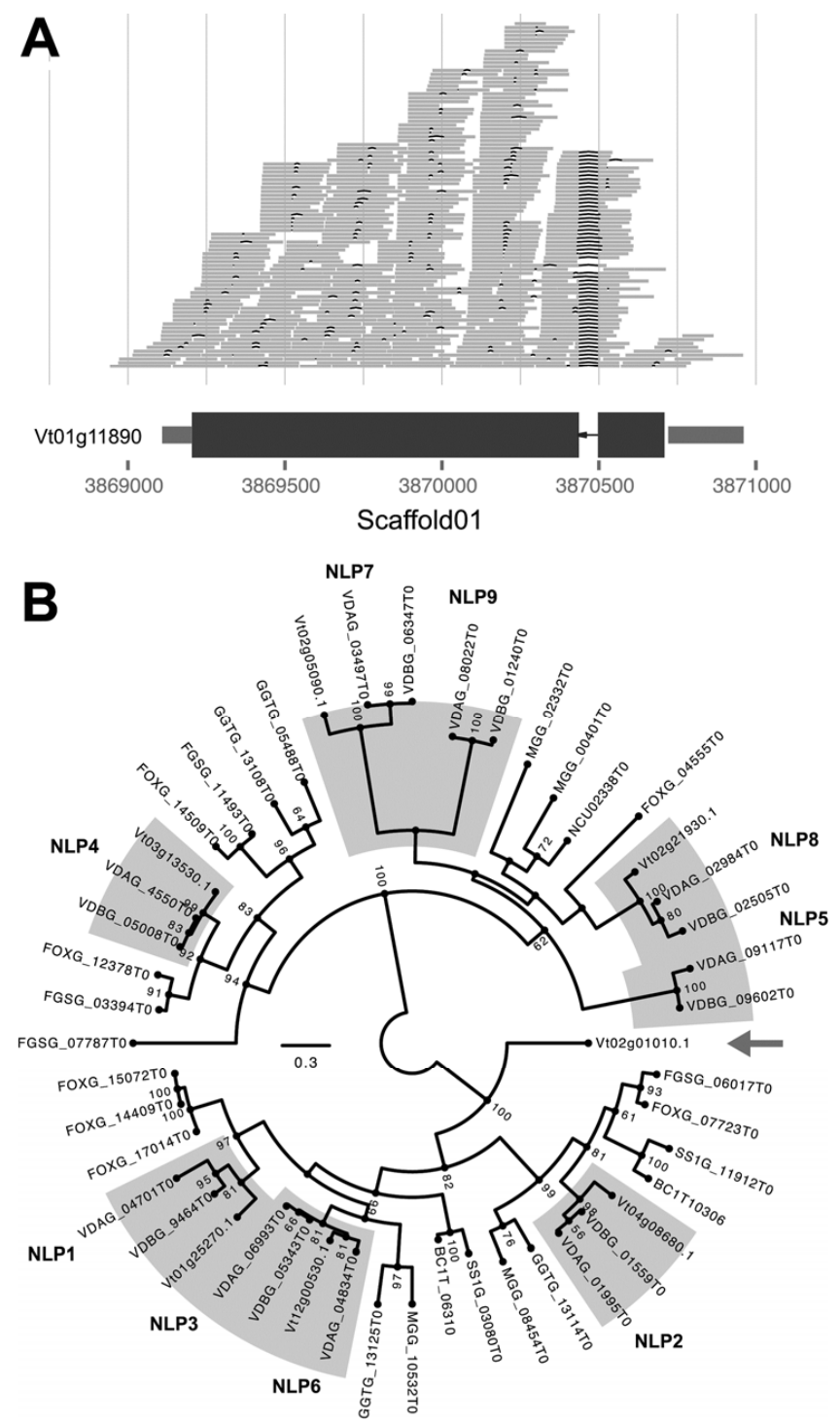

Fig. 7. The genome of Verticillium tricorpus encodes effector candidates similar to those encoded by pathogenic Verticillium spp. A, RNA-Seq expression of a gene encoding the LysM effector Vt01g11890. B, Phylogenetic relationship of necrosis and ethylene inducing-like (NLP) in related filamentous fungi. Orthologous groups of previously described NLP classes in Verticillium are highlighted in gray (Santhanam et al. 2013) and the single highly expressed NLP (Vt02g01010) in V. tricorpus is indicated by an arrow. 
expressed during infection and acts as a virulence factor on tomato (de Jonge et al. 2013). So far, the role of the remaining four core LysM effectors during plant infection could not be demonstrated and their function remains enigmatic (Kombrink and Thomma 2013). Rather than a role in pathogenicity, Verticillium LysM effectors could be involved in protection from competing microbes in the soil (de Jonge and Thomma 2009; Kombrink and Thomma 2013; Rovenich et al. 2014).

Similar to LysM effectors, necrosis and ethylene inducinglike proteins (NLP) also are widespread and occur in organisms with diverse life styles (Gijzen and Nürnberger 2006). Even though they have been observed in both pathogenic and saprophytic species, e.g., Neurospora crassa (Fig. 7B), they have gained considerable attention in pathogenic species (Gijzen and Nürnberger 2006). Many fungi contain few (one or two) NLP, while the NLP family is expanded in several pathogenic oomycetes and particular fungi (Gijzen and Nürnberger 2006; Santhanam et al. 2013; Seidl et al. 2011), including V. dahliae and $V$. alfalfae, that, depending on the strain, contain either seven or eight members (Santhanam et al. 2013). Recent analyses revealed that only two of the $V$. dahliae NLP, NLP1 and NLP2, induce cell death and are required for virulence on tomato and Arabidopsis (Santhanam et al. 2013) while the role of the remaining, noncytolytic NLP remains unclear. Interestingly, VdNLP1 also plays a role in vegetative growth and asexual production, hinting to alternative functions of NLP besides their necrotic activity (Santhanam et al. 2013). An initial survey of $V$. tricorpus prior to the completion of the genome assembly identified only four NLP homologs (Santhanam et al. 2013). However, the presently assembled genome of $V$. tricorpus contains seven NLP genes, of which six are clear orthologs of previously described $V$. dahliae NLP (Fig. 7B). Interestingly, $V$. tricorpus encodes orthologs of NLP1 and NLP2. Both of these orthologs were expressed at low levels (7.8 and 15 read counts per $1 \mathrm{~kb}$, respectively), in contrast to a $V$. tricorpus-specific NLP homolog (Vt02g01010) that was highly expressed in the tomato cell suspension (368.7 read counts per $1 \mathrm{~kb}$ ). Thus, presently the function of $V$. tricorpus NLP remains enigmatic.

In summary, we revealed an effector repertoire in $V$. tricorpus that is surprisingly similarly composed when compared with its pathogenic relatives. Members of some of the identified effector families are commonly observed in nonpathogenic species such as saprophytes and, thus, do not necessarily represent genuine pathogenicity factors. Moreover, homologs of $V$. dahliae effector genes, such as $N L P 2$, are not always identical in $V$. tricorpus and may, thus, have diverged to different functions. Furthermore, a considerable number of SSCP do not display significant similarity to any known (effector) family, and several of these uncharacterized proteins might play important roles in niche establishment and competition with other microbes. For example, $V$. tricorpus has recently been shown to be an antagonist of $V$. dahliae on lettuce and artichoke (Qin et al. 2008) and SSCP of unknown function might be involved in this interaction. Thus, the genome assembly of $V$. tricorpus presents the foundation for the identification and initial characterization of effector families. Realistically, however, the functions of most effector families and their members remain unknown and need experimental characterization.

\section{Conclusions.}

In the last decade, rapid technological advances made genomic sequencing a commodity that provides a plethora of genomic sequences. Many of these genomes are from closely related species that differ in their ecological niches. We anticipated that a high-quality genomic sequence of the saprophyte $V$. tricorpus would shed light on the origin of pathogenicity within the genus Verticillium. However, contrary to our expec- tations, the genome of $V$. tricorpus contains many of the hallmarks that also characterize pathogenic Verticillium spp. In particular, $V$. tricorpus encodes an effector repertoire that largely resembles that of its pathogenic relatives. Assuming that pathogenicity in fungi evolved from saprophytes, alternative roles for effectors, e.g., during interactions with other microorganisms that are encountered in the same niche, seem likely (Rovenich et al. 2014). During the evolution of pathogenicity, these proteins might have acquired altered functionalities that facilitate the pathogenic lifestyle. However, a distinct difference in the repetitive element content between $\mathrm{V}$. tricorpus and its pathogenic relatives may suggest that repeats are involved in the evolution of pathogenicity in the Verticillium genus. We anticipate that further comparative genomics together with functional characterization of effector proteins in species with different lifestyles will enhance our understanding of their role in facilitating a pathogenic lifestyle.

\section{MATERIALS AND METHODS}

\section{Plant inoculations.}

All plant inoculations were performed on 10-day-old tomato seedlings (cv. MoneyMaker) using root-dip inoculation as described previously (Fradin et al. 2009). Briefly, seedlings were uprooted and inoculated by dipping the roots for $2 \mathrm{~min}$ in a conidial suspension $\left(10^{6}\right.$ conidia per milliliter) in water. After replanting in soil, plants were incubated at standard greenhouse conditions of a 16-h-light and 8-h-dark regime and $60 \%$ relative humidity. Disease progression was monitored for 15 days after inoculation. To determine in planta colonization, stem sections at the height of the first internode were taken, surfacesterilized, and sliced. The slices were placed on potato dextrose agar supplemented with chloramphenicol at $50 \mu \mathrm{g} / \mathrm{ml}$ and were incubated at $22^{\circ} \mathrm{C}$ for 10 days. For in planta biomass quantification, stems of three inoculated plants were harvested at 15 days after inoculation. The samples were ground to powder and genomic DNA was isolated. Real-time PCR on genomic DNA was carried out and was analyzed using the 7300 SDS software (Applied Biosystems). A Verticillium-specific primer pair was used to quantify fungal colonization (5'-CATTGCCC AAGTTTACCTCC-3' combined with 5'-GCCAGCGTGTCTA TCTTCTC-3').

\section{DNA extraction.}

DNA was isolated from conidia and mycelium fragments that were harvested from 10-day-old cultures grown in liquid potato dextrose broth at $28^{\circ} \mathrm{C}$. DNA isolation was performed according to the following protocol. First, mycelium was scraped directly from the surface of an agar plate, was suspended in water, and was transferred to a 50-ml tube. The tube was vortexed briefly and was centrifuged at maximum speed for $10 \mathrm{~min}$. After centrifugation, $0.5 \mathrm{ml}$ of $0.1 \mathrm{M}$ Tris $\mathrm{HCl}(\mathrm{pH} 8)$ was added to the pellet, which was mixed and subsequently transferred to a 2-ml screw-cap tube. Three metal beads were added and the tube was shaken in a bead beater at the highest speed for $45 \mathrm{~s}$. After shaking, $1 \mathrm{ml}$ of phenol/chloroform/isoamylalcohol (24:24:1) was added. The tube was mixed and centrifuged at maximum speed for $7 \mathrm{~min}$, and then, the water phase $(400 \mu \mathrm{l})$ was transferred to a new tube. In the new tube, $800 \mu$ l of isopropanol was added and the tube was centrifuged, after mixing at maximum speed for $20 \mathrm{~min}$ in the cold room. After centrifugation, the isopropanol was removed and the DNA pellet was air-dried. Subsequently, the pellet was dissolved in $50 \mu \mathrm{l}$ of deionized $\mathrm{H}_{2} \mathrm{O}$.

\section{Genome sequencing and assembly.}

Two sequence libraries (500-bp and 5-kb insert size) of $V$. tricorpus were prepared and paired ends were sequenced at the 
Beijing Genome Institute (BGI), using the Illumina highthroughput sequencing platform. In addition, a long-insert library $(10 \mathrm{~kb})$ was generated and sequenced with SMRT sequencing technology on a PacBio platform at BaseClear (Leiden, The Netherlands). Genome assembly was performed as described previously (Faino and Thomma 2014). Contiguity of scaffolds and the correct placement of contigs were manually assessed. To this end, we manually inspected paired-end reads for concordant mapping and coverage of gapped regions between contigs. Genome statistics were calculated using QUAST (Gurevich et al. 2013). Repeats were identified and characterized using the de novo repeat identification with RepeatModeler (Smit and Hubley 2008) (1.0.7; default setting). Subsequently, de novo-identified repeats were combined with the repeat library from RepBase (Jurka et al. 2005) (release 31-04-2014) to identify and annotate families of repetitive elements, using RepeatMasker (Smit et al. 1996) (open-4.0.5) in sensitive mode.

\section{Karyotyping.}

Karyotyping was performed as outlined by de Jonge and associates (2013). Briefly, V. tricorpus protoplasts were prepared as described by Mehrabi and colleagues (2012). Karyotyping was performed using a CHEF Mapper XA pulsed field electrophoresis system (Bio-Rad), using the auto algorithm function with low- and high-molecular weight setting at 2 and $6 \mathrm{Mb}$, respectively. Chromosomes were separated in $0.8 \%$ low EEO agarose gel (US Biologicals). Two chromosome size markers (Bio-Rad) were loaded as a reference marker. After electrophoresis, the gel was stained with ethidium bromide $(1 \mu \mathrm{g} / \mathrm{ml})$ in water for $1 \mathrm{~h}$ and was de-stained in water for $2 \mathrm{~h}$.

\section{Gene prediction and annotation.}

For RNA sequencing, V. tricorpus was cultured in a 10-dayold tomato cell suspension for 2 days. RNA was isolated based on Trizol RNA extraction (Simms et al., 1993). An RNA-Seq library (180 bp insert size) was prepared and sequenced (100bp reads) at BGI. The RNA-Seq reads were mapped onto the draft V. tricorpus genome using Tophat (Trapnell et al. 2009) and PASA (Haas et al. 2003). Oases (Schulz et al. 2012) and Trinity (Grabherr et al. 2011) were used to assemble the RNASeq data independently from the genome assembly. The assembled transcripts derived from Oases, Trinity, and PASA were combined and used as cDNA expressed sequence tag evidence in Maker2 (Holt and Yandell 2011). Moreover, assembled RNA-Seq reads from $V$. dahliae were used as evidence for gene prediction as well as protein sequences from 35 different fungal proteomes. The annotation of the predicted genes was done by BLASTp (Altschul et al. 1990) searches to the National Center for Biotechnology Information (NCBI) nonredundant database and by InterProScan (Jones et al. 2014). The BLASTp analysis and the InterProScan were merged together using Blast2GO (Conesa et al. 2005).

To estimate gene expression levels in V. tricorpus, RNA-Seq reads were mapped to the draft genome of $V$. tricorpus using TopHat (Trapnell et al. 2009). Subsequently, the number of reads that were mapped to each gene were determined and normalized for the length of the transcript (number of reads per $1 \mathrm{~kb}$ of transcript). Similarly, gene expression in $V$. dahliae JR2 was estimated using previously generated RNA-Seq data (de Jonge et al. 2012). For each condition, the reads were mapped to the draft genome assembly of $V$. dahliae JR2 using TopHat (Trapnell et al. 2009) and were summarized as reads per kilobase of transcript per million of mapped reads.

Secreted proteins were predicted using a combination of SignalP3 (Dyrløv Bendtsen et al. 2004), SignalP4 (Petersen et al. 2011), TargetP (Emanuelsson et al. 2000), and TMHMM
(Krogh et al. 2001). Proteins showing positive predictions from i) both predictors (HMM and neural network, default settings) of SignalP3, ii) SignalP4 (default settings), and iii) TargetP (predicted localization "secreted"; default settings) were considered as candidate effectors. Moreover, we filtered this list by removing proteins that contained i) more than a single TMHMM predicted transmembrane domain or ii) proteins with a single TMHMM predicted transmembrane domain, if this transmembrane domain starts outside of the first 35 amino acids or overlaps less than 10 amino acids with the predicted signal peptide. Protein domains observed in candidate effectors proteins, e.g., NLP and LysMs, were identified by mining the InterProScan annotations.

\section{Comparative genomics.}

The species phylogeny was based on the amino acid sequences of 130 single-copy gene families that were identified in the predicted proteomes of $V$. tricorpus and nine related fungi using the CEGMA pipeline (Parra et al. 2007). Individual families were aligned using mafft (LINSi; v7.047b) (Katoh et al. 2002) and were subsequently concatenated. Maximum likelihood phylogeny was inferred using RAxML (v7.6.3) (Stamatakis 2006; Stamatakis et al. 2008) with the GAMMA model of rate heterogeneity and the Whelan and Goldman (WAG) model of amino acid substitutions. The robustness of the inferred phylogeny was assessed by 1,000 rapid bootstrap approximations.

The phylogeny of the NLP was based on the identified NLP domain (PF05630, excluding short fragments) using InterProScan (Jones et al. 2014) of 10 filamentous fungi. Subsequently, the NLP domain was excised, aligned with mafft (LINSi; v7.047b) (Katoh et al. 2002). Maximum likelihood phylogeny was inferred using RAxML (v7.6.3) (Stamatakis 2006; Stamatakis et al. 2008) with the GAMMA model of rate heterogeneity and the WAG model of amino acid substitutions. The robustness of the inferred phylogeny was assessed by 1,000 rapid bootstrap approximations.

We built families of gene-encoding secreted proteins in the analyzed Verticillium spp. and seven related fungi using OrthoMCL (default setting) (Li et al. 2003). Beforehand, similarity between proteins was established by an all-vs.-all analyses using BLASTp (E-value cutoff 1e-5, soft filtering) (Altschul et al. 1990, 1997). The ancestral set of families and the gains and losses were inferred by reconciliation of the binary presence or absence profiles of members constituting an orthologous group with the species phylogeny using DOLLOP (Felsenstein 2002), assuming the irreversibility of the loss an orthologous group, i.e., an orthologous group can only be gained, however individual members can be lost multiple times independently. To calculate the value of $\mathrm{K}_{\mathrm{S}}, \mathrm{K}_{\mathrm{A}}$, and the $\mathrm{K}_{\mathrm{A}} / \mathrm{K}_{\mathrm{S}}$ ratio, we extracted sequences of the four analyzed Verticillium strains from the previously determined OrthoMCL families. Subsequently, we aligned the coding sequences per gene family guided by protein alignment and determined the pairwise $K_{S}$, $\mathrm{K}_{\mathrm{A}}$, and $\mathrm{K}_{\mathrm{A}} / \mathrm{K}_{\mathrm{S}}$ ratios using the 'kaks' function of the 'seqinr' package. For further analysis, only genes with $\mathrm{K}_{\mathrm{A}}$ or $\mathrm{K}_{\mathrm{S}}$ value $\leq$ 5 were considered, to avoid saturation effects. Similarly, we built OrthoMCL families for all genes of the four Verticillium spp. and determined $\mathrm{K}_{\mathrm{A}}, \mathrm{K}_{\mathrm{S}}$, and $\mathrm{K}_{\mathrm{A}} / \mathrm{K}_{\mathrm{S}}$ ratios as outlined above.

To identify clustering of genes that encode secreted proteins, we scanned each scaffold with CROC (Pignatelli et al. 2009). Briefly, CROC scans each scaffold, represented as a string of genes, with a sliding window (number of genes per window = 20; slide per window $=1$; minimum of five genes of interest per window). For each window, the probability to observe that many (or more) genes encoding secreted genes by chance is assessed using a hypergeometric distribution test (Pignatelli et 
al. 2009). Subsequently, multiple-testing correction using Benjamini-Hochberg was applied. Transmembrane localized proteins were predicted using TMHMM (Krogh et al. 2001), and nuclear localized proteins were predicted, using a combination of WoLF PSORT (Horton et al. 2007) and NLStradamus (Nguyen Ba et al. 2009), and clustering was a subsequently assessed as described above.

\section{Data access.}

The genome assembly has been associated with the NCBI GenBank BioProject PRJNA229139 and the BioSample SAMN02415140. The assembly is deposited in the DDBJ/EMBL/GenBank database under the accession JPET00000000. The raw RNA-Seq sequence reads have been associated with the NCBI Sequence Read Archive under the same BioProject and BioSample numbers and is deposited under the accession SRX658508.

\section{ACKNOWLEDGMENTS}

The authors acknowledge support by the Research Council Earth and Life Sciences (ALW) of the Netherlands Organization of Scientific Research (NWO). Moreover, we thank B. Essenstam for excellent plant care and X. Wang (USDA-ARS) for excellent technical assistance.

\section{LITERATURE CITED}

Altschul, S. F., Gish, W., Miller, W., Myers, E. W., and Lipman, D. J. 1990. Basic local alignment search tool. J. Mol. Biol. 215:403-410.

Altschul, S. F., Madden, T. L., Schäffer, A. A., Zhang, J., Zhang, Z., Miller, W., and Lipman, D. J. 1997. Gapped BLAST and PSI-BLAST: A new generation of protein database search programs. Nucleic Acids Res. 25:3389-3402.

Bolton, M. D., van Esse, H. P., Vossen, J. H., de Jonge, R., Stergiopoulos, I., Stulemeijer, I. J. E., van den Berg, G. C. M., Borrás-Hidalgo, O., Dekker, H. L., de Koster, C. G., de Wit, P. J. G. M., Joosten, M. H. A. J., and Thomma, B. P. H. J. 2008. The novel Cladosporium fulvum lysin motif effector Ecp6 is a virulence factor with orthologues in other fungal species. Mol. Microbiol. 69:119-136.

Brown, N. A., Antoniw, J., and Hammond-Kosack, K. E. 2012. The predicted secretome of the plant pathogenic fungus Fusarium graminearum: A refined comparative analysis. PLoS ONE 7:e33731. Published online.

Choi, J., Park, J., Kim, D., Jung, K., Kang, S., and Lee, Y.-H. 2010. Fungal secretome database: Integrated platform for annotation of fungal secretomes. BMC Genomics 11:105.

Conesa, A., Gotz, S., Garcia-Gomez, J. M., Terol, J., Talon, M., and Robles, M. 2005. Blast2GO: A universal tool for annotation, visualization and analysis in functional genomics research. Bioinformatics 21:3674-3676.

de Jonge, R., and Thomma, B. P. H. J. 2009. Fungal LysM effectors: Extinguishers of host immunity? Trends Microbiol. 17:151-157.

de Jonge, R., Peter van Esse, H., Kombrink, A., Shinya, T., Desaki, Y., Bours, R., van der Krol, S., Shibuya, N., Joosten, M. H. A. J., and Thomma, B. P. H. J. 2010. Conserved fungal LysM effector Ecp6 prevents chitin-triggered immunity in plants. Science 329:953-955.

de Jonge, R., Bolton, M. D., and Thomma, B. P. 2011. How filamentous pathogens co-opt plants: The ins and outs of fungal effectors. Curr. Opin. Plant Biol. 14:400-406.

de Jonge, R., Peter van Esse, H., Maruthachalam, K., Bolton, M. D., Santhanam, P., Saber, M. K., Zhang, Z., Usami, T., Lievens, B., Subbarao, K. V., and Thomma, B. P. H. J. 2012. Tomato immune receptor Ve1 recognizes effector of multiple fungal pathogens uncovered by genome and RNA sequencing. Proc. Natl. Acad. Sci. U.S.A. 109:5110-5115.

de Jonge, R., Bolton, M. D., Kombrink, A., van den Berg, G. C. M., Yadeta, K. A., and Thomma, B. P. H. J. 2013. Extensive chromosomal reshuffling drives evolution of virulence in an asexual pathogen. Genome Res. 23:1271-1282.

Dyrløv Bendtsen, J., Nielsen, H., Heijne, von, G., and Brunak, S. 2004. Improved prediction of signal peptides: SignalP 3.0. J. Mol. Biol. 340:783-795.

Emanuelsson, O., Nielsen, H., Brunak, S., and Heijne, von, G. 2000. Predicting subcellular localization of proteins based on their N-terminal amino acid sequence. J. Mol. Biol. 300:1005-1016.

Faino, L., and Thomma, B. P. H. J. 2014. Get your high-quality low-cost genome sequence. Trends Plant Sci. 19:288-291.

Felsenstein, J. 2002. PHYLIP (Phylogeny Inference Package) version 3.6a3. Department of Genome Sciences, University of Washington, Seattle.

Fradin, E. F., and Thomma, B. P. H. J. 2006. Physiology and molecular aspects of Verticillium wilt diseases caused by $V$. dahliae and V. alboatrum. Mol. Plant Pathol. 7:71-86.

Fradin, E. F., Zhang, Z., Juarez Ayala, J. C., Castroverde, C. D. M., Nazar, R. N., Robb, J., Liu, C.-M., and Thomma, B. P. H. J. 2009. Genetic dissection of Verticillium wilt resistance mediated by tomato Ve1. Plant Physiol. 150:320-332.

Fulnecková, J., Sevcíková, T., Fajkus, J., Lukesová, A., Lukes, M., Vlcek, C., Lang, B. F., Kim, E., Elias, M., and Sykorová, E. 2013. A broad phylogenetic survey unveils the diversity and evolution of telomeres in eukaryotes. Genome Biol Evol. 5:468-483.

Gaderer, R., Bonazza, K., and Seidl-Seiboth, V. 2014. Cerato-platanins: A fungal protein family with intriguing properties and application potential. Appl. Microbiol. Biotechnol. 98:4795-4803.

Gijzen, M., and Nürnberger, T. 2006. Nep1-like proteins from plant pathogens: Recruitment and diversification of the NPP1 domain across taxa. Phytochemistry. 67:1800-1807.

Gnerre, S., Maccallum, I., Przybylski, D., Ribeiro, F. J., Burton, J. N., Walker, B. J., Sharpe, T., Hall, G., Shea, T. P., Sykes, S., Berlin, A. M., Aird, D., Costello, M., Daza, R., Williams, L., Nicol, R., Gnirke, A., Nusbaum, C., Lander, E. S., and Jaffe, D. B. 2011. High-quality draft assemblies of mammalian genomes from massively parallel sequence data. Proc. Natl. Acad. Sci. U.S.A. 108:1513-1518.

Grabherr, M. G., Haas, B. J., Yassour, M., Levin, J. Z., Thompson, D. A., Amit, I., Adiconis, X., Fan, L., Raychowdhury, R., Zeng, Q., Chen, Z., Mauceli, E., Hacohen, N., Gnirke, A., Rhind, N., di Palma, F., Birren, B. W., Nusbaum, C., Lindblad-Toh, K., Friedman, N., and Regev, A. 2011. Full-length transcriptome assembly from RNA-Seq data without a reference genome. Nat. Biotechnol. 29:644-652.

Gurevich, A., Saveliev, V., Vyahhi, N., and Tesler, G. 2013. QUAST: Quality assessment tool for genome assemblies. Bioinformatics 29:10721075.

Guyon, K., Balagué, C., Roby, D., and Raffaele, S. 2014. Secretome analysis reveals effector candidates associated with broad host range necrotrophy in the fungal plant pathogen Sclerotinia sclerotiorum. BMC Genomics 15:336.

Haas, B. J., Delcher, A. L., Mount, S. M., Wortman, J. R., Smith, R. K., Hannick, L. I., Maiti, R., Ronning, C. M., Rusch, D. B., Town, C. D., Salzberg, S. L., and White, O. 2003. Improving the Arabidopsis genome annotation using maximal transcript alignment assemblies. Nucleic Acids Res. 31:5654-5666.

Holt, C., and Yandell, M. 2011. MAKER2: An annotation pipeline and genome-database management tool for second-generation genome projects. BMC Bioinformatics 12:491.

Horton, P., Park, K.-J., Obayashi, T., Fujita, N., Harada, H., Adams-Collier, C. J., and Nakai, K. 2007. WoLF PSORT: Protein localization predictor. Nucleic Acids Res. 35:W585-7.

Inderbitzin, P., and Subbarao, K. V. 2014. Verticillium systematics and evolution: How confusion impedes verticillium wilt management and how to resolve it. Phytopathology 104:564-574.

Inderbitzin, P., Bostock, R. M., Davis, R. M., Usami, T., Platt, H. W., and Subbarao, K. V. 2011. Phylogenetics and taxonomy of the fungal vascular wilt pathogen Verticillium, with the descriptions of five new species. A. Idnurm, ed. PLoS ONE 6:e28341. Published online.

Isaac, I. 1956. Some soil factors affecting Verticillium wilt of antirrhinum. Annals of Applied Biology. 44:105-112.

Isaac, I. 1967. Speciation in Verticillium. Annu. Rev. Phytopathol. 5:201222.

Jones, P., Binns, D., Chang, H.-Y., Fraser, M., Li, W., McAnulla, C., McWilliam, H., Maslen, J., Mitchell, A., Nuka, G., Pesseat, S., Quinn, A. F., Sangrador-Vegas, A., Scheremetjew, M., Yong, S.-Y., Lopez, R., and Hunter, S. 2014. InterProScan 5: Genome-scale protein function classification. Bioinformatics 30:1236-1240.

Jurka, J., Kapitonov, V. V., Pavlicek, A., Klonowski, P., Kohany, O., and Walichiewicz, J. 2005. Repbase Update, a database of eukaryotic repetitive elements. Cytogenet. Genome Res. 110:462-467.

Katoh, K., Misawa, K., Kumar, S., and Miyata, T. 2002. MAFFT: A novel method for rapid multiple sequence alignment based on fast Fourier transform. Nucleic Acids Res. 30:3059-3066.

Kim, D., Pertea, G., Trapnell, C., Pimentel, H., Kelley, R., and Salzberg, S. L. 2013. TopHat2: Accurate alignment of transcriptomes in the presence of insertions, deletions and gene fusions. Genome Biol. 14:R36.

Klimes, A., Amyotte, S. G., Grant, S., Kang, S., and Dobinson, K. F. 2008 Microsclerotia development in Verticillium dahliae: Regulation and differential expression of the hydrophobin gene VDH1. Fungal Genet. Biol. 45:1525-1532.

Klosterman, S. J., Atallah, Z. K., Vallad, G. E., and Subbarao, K. V. 2009. Diversity, pathogenicity, and management of Verticillium species. Annu. 
Rev. Phytopathol. 47:39-62.

Klosterman, S. J., Subbarao, K. V., Kang, S., Veronese, P., Gold, S. E., Thomma, B. P. H. J., Chen, Z., Henrissat, B., Lee, Y.-H., Park, J., GarciaPedrajas, M. D., Barbara, D. J., Anchieta, A., de Jonge, R., Santhanam, P., Maruthachalam, K., Atallah, Z., Amyotte, S. G., Paz, Z., Inderbitzin, P., Hayes, R. J., Heiman, D. I., Young, S., Zeng, Q., Engels, R., Galagan, J., Cuomo, C. A., Dobinson, K. F., and Ma, L.-J. 2011. Comparative genomics yields insights into niche adaptation of plant vascular wilt pathogens. J.L. Dangl, ed. PLoS Pathog. 7:e1002137. Published online.

Kombrink, A. 2014. Functional analysis of LysM effectors secreted by fungal plant pathogens. PhD thesis. Wageningen University, The Netherlands.

Kombrink, A., and Thomma, B. P. H. J. 2013. LysM effectors: Secreted proteins supporting fungal life. PLoS Pathog. 9:e1003769. Published online.

Koren, S., Schatz, M. C., Walenz, B. P., Martin, J., Howard, J. T., Ganapathy, G., Wang, Z., Rasko, D. A., McCombie, W. R., Jarvis, E. D., and Adam M Phillippy. 2012. Hybrid error correction and de novo assembly of single-molecule sequencing reads. Nat. Biotechnol. 30:693-700.

Korf, I. 2004. Gene finding in novel genomes. BMC Bioinformatics 5:59.

Krogh, A., Larsson, B., Heijne, von, G., and Sonnhammer, E. L. 2001. Predicting transmembrane protein topology with a hidden Markov model: Application to complete genomes. J. Mol. Biol. 305:567-580.

Li, L., Stoeckert, C. J., and Roos, D. S. 2003. OrthoMCL: Identification of ortholog groups for eukaryotic genomes. Genome Res. 13:2178-2189.

Lukashin, A. V., and Borodovsky, M. 1998. GeneMark.hmm: New solutions for gene finding. Nucleic Acids Res. 26:1107-1115.

Marshall, R., Kombrink, A., Motteram, J., Loza-Reyes, E., Lucas, J., Hammond-Kosack, K. E., Thomma, B. P. H. J., and Rudd, J. J. 2011. Analysis of two in planta expressed LysM effector homologs from the fungus Mycosphaerella graminicola reveals novel functional properties and varying contributions to virulence on wheat. Plant Physiol. 156:756-769.

Mehrabi, R., Taga, M., Aghaee, M., de Wit, P. J. G. M., and Kema, G. H. J 2012. Karyotyping methods for fungi. Methods Mol. Biol. 835:591602

Mentlak, T. A., Kombrink, A., Shinya, T., Ryder, L. S., Otomo, I., Saitoh, H., Terauchi, R., Nishizawa, Y., Shibuya, N., Thomma, B. P. H. J., and Talbot, N. J. 2012. Effector-mediated suppression of chitin-triggered immunity by Magnaporthe oryzae is necessary for rice blast disease. Plant Cell 24:322-335.

Metzker, M. L. 2010. Sequencing technologies-The next generation. Nat. Rev. Genet. 11:31-46.

Nguyen Ba, A. N., Pogoutse, A., Provart, N., and Moses, A. M. 2009. NLStradamus: A simple Hidden Markov Model for nuclear localization signal prediction. BMC Bioinformatics 10:202.

Parra, G., Bradnam, K., and Korf, I. 2007. CEGMA: A pipeline to accurately annotate core genes in eukaryotic genomes. Bioinformatics 23:1061-1067.

Parra, G., Bradnam, K., Ning, Z., Keane, T., and Korf, I. 2009. Assessing the gene space in draft genomes. Nucleic Acids Res. 37:289-297.

Petersen, T. N., Brunak, S., Heijne, von, G., and Nielsen, H. 2011. SignalP 4.0: Discriminating signal peptides from transmembrane regions. Nat. Methods 8:785-786.

Pignatelli, M., Serras, F., Moya, A., Guigó, R., and Corominas, M. 2009. CROC: Finding chromosomal clusters in eukaryotic genomes. Bioinformatics 25:1552-1553.

Powell, M., Gundersen, B., Miles, C., Coats, K., and Inglis, D. A. 2013. First report of Verticillium wilt on lettuce (Lactuca sativa) in Washington caused by Verticillium tricorpus. Plant Dis. 97:996-996.

Qin, Q. M., Qin, Q.-M., Vallad, G. E., Vallad, G. E., Subbarao, K. V., and Subbarao, K. V. 2008. Characterization of Verticillium dahliae and V. tricorpus isolates from lettuce and artichoke. Plant Dis. 92:69-77.

Raffaele, S., and Kamoun, S. 2012. Genome evolution in filamentous plant pathogens: Why bigger can be better. Nat. Rev. Microbiol. 10:417-430.

Raffaele, S., Farrer, R. A., Cano, L. M., Studholme, D. J., MacLean, D., Thines, M., Jiang, R. H. Y., Zody, M. C., Kunjeti, S. G., Donofrio, N. M., Meyers, B. C., Nusbaum, C., and Kamoun, S. 2010. Genome evolu- tion following host jumps in the Irish potato famine pathogen lineage. Science 330:1540-1543.

Rovenich, H., Boshoven, J. C., and Thomma, B. P. 2014. Filamentous pathogen effector functions: Of pathogens, hosts and microbiomes. Curr. Opin. Plant Biol. 20C:96-103.

Santhanam, P., van Esse, H. P., Albert, I., Faino, L., Nürnberger, T., and Thomma, B. P. H. J. 2013. Evidence for functional diversification within a fungal NEP1-like protein family. Mol. Plant-Microbe Interact. 26:278-286.

Saunders, D. G. O., Win, J., Cano, L. M., Szabo, L. J., Kamoun, S., and Raffaele, S. 2012. Using hierarchical clustering of secreted protein families to classify and rank candidate effectors of rust fungi. J.E. Stajich, ed. PLoS ONE 7:e29847. Published online.

Sánchez-Vallet, A., Saleem-Batcha, R., Kombrink, A., Hansen, G., Valkenburg, D.-J., Thomma, B. P., and Mesters, J. R. 2013. Fungal effector Ecp6 outcompetes host immune receptor for chitin binding through intrachain LysM dimerization. Elife 2:e00790. Published online.

Schadt, E. E., Turner, S., and Kasarskis, A. 2010. A window into thirdgeneration sequencing. Hum. Mol. Genet. 19:R227-40.

Schulz, M. H., Zerbino, D. R., Vingron, M., and Birney, E. 2012. Oases: Robust de novo RNA-seq assembly across the dynamic range of expression levels. Bioinformatics 28:1086-1092.

Seidl, M. F., and Thomma, B. P. H. J. 2014. Sex or no sex: Evolutionary adaptation occurs regardless. Bioessays 36:335-345.

Seidl, M. F., Van den Ackerveken, G., Govers, F., and Snel, B. 2011. A domain-centric analysis of oomycete plant pathogen genomes reveals unique protein organization. Plant Physiol. 155:628-644.

Smit, A. F. A., and Hubley, R. 2008. RepeatModeler Open-1.0.

Smit, A. F. A., Hubley, R., and Green, P. 1996. RepeatMasker Open-3.0.

Stamatakis, A. 2006. RAxML-VI-HPC: Maximum likelihood-based phylogenetic analyses with thousands of taxa and mixed models. Bioinformatics 22:2688-2690.

Stamatakis, A., Hoover, P., and Rougemont, J. 2008. A rapid bootstrap algorithm for the RAxML Web servers. Syst. Biol. 57:758-771.

Stanke, M., Steinkamp, R., Waack, S., and Morgenstern, B. 2004. AUGUSTUS: A web server for gene finding in eukaryotes. Nucleic Acids Res. 32:W309-12.

Talbot, N. J., Ebbole, D. J., and Hamer, J. E. 1993. Identification and characterization of MPG1, a gene involved in pathogenicity from the rice blast fungus Magnaporthe grisea. Plant Cell 5:1575-1590.

Thomma, B. P. H. J., Nurnberger, T., and Joosten, M. H. A. J. 2011. Of PAMPs and effectors: The blurred PTI-ETI dichotomy. Plant Cell 23:415

Trapnell, C., Pachter, L., and Salzberg, S. L. 2009. TopHat: Discovering splice junctions with RNA-Seq. Bioinformatics 25:1105-1111.

Tritt, A., Eisen, J. A., Facciotti, M. T., and Darling, A. E. 2012. An integrated pipeline for de novo assembly of microbial genomes. PLoS ONE 7:e42304. Published online.

Wicker, T., Oberhaensli, S., Parlange, F., Buchmann, J. P., Shatalina, M., Roffler, S., Ben-David, R., Doležel, J., Simková, H., Schulze-Lefert, P., Spanu, P. D., Bruggmann, R., Amselem, J., Quesneville, H., Ver Loren van Themaat, E., Paape, T., Shimizu, K. K., and Keller, B. 2013. The wheat powdery mildew genome shows the unique evolution of an obligate biotroph. Nat. Genet. 45:1092-1096.

Yin, Y., Mao, X., Yang, J., Chen, X., Mao, F., and Xu, Y. 2012. dbCAN: A web resource for automated carbohydrate-active enzyme annotation. Nucleic Acids Res. 40:W445-51.

Zdobnov, E. M., and Apweiler, R. 2001. InterProScan--an integration platform for the signature-recognition methods in InterPro. Bioinformatics 17:847-848.

Zhao, Z., Liu, H., Wang, C., and Xu, J.-R. 2013. Comparative analysis of fungal genomes reveals different plant cell wall degrading capacity in fungi. BMC Genomics 14:274.

\section{AUTHOR-RECOMMENDED INTERNET RESOURCE}

The $\mathrm{R}$ project seqinr package:

cran.r-project.org/web/packages/seqinr/index.htm 